\title{
No effect of gamification on attrition from a longitudinal cognitive testing study
}

Jim Lumsden ${ }^{1,2}$, Andy Skinner ${ }^{1,2}$, David Coyle ${ }^{3}$, Natalia Lawrence ${ }^{4}$, Marcus Munafó ${ }^{1,2}$

${ }^{1}$ MRC Integrative Epidemiology Unit (IEU), University of Bristol, Bristol, United Kingdom

${ }^{2}$ School of Experimental Psychology, University of Bristol, Bristol, United Kingdom

${ }^{3}$ School of Computer Science, University College Dublin, Dublin, Ireland

${ }^{4}$ School of Psychology,

College of Life and Environmental Sciences, University of Exeter, Exeter, United Kingdom

* Corresponding author

E-mail: jim.lumsden@bristol.ac.uk

Telephone: $+44(0) 1179288450$

Address: Jim Lumsden, 12a Priory Rd, School of Experimental Psychology, University of Bristol, BS8 1TU, UK 


\begin{abstract}
The prospect of assessing cognition longitudinally is attractive to researchers, health practitioners and pharmaceutical companies alike. However, such repeated-testing regimes place a considerable burden on participants, and with cognitive tasks typically being regarded as effortful and unengaging, these studies may experience high levels of participant attrition. One potential solution is to gamify these tasks to make them more engaging: increasing participant willingness to take part and reducing attrition. However, such an approach must balance task validity with introducing entertaining gamelike elements.

We investigated the effects of gamelike features on participant attrition using a betweensubjects, longitudinal online testing study. We used three variants of a common cognitive task, the stop signal task, with a single gamelike feature in each: one variant where points were rewarded for performing optimally, another where the task was given a graphical theme, and a third variant which was a standard stop signal task and served as a control condition. Participants completed four compulsory test sessions over four consecutive days before entering a six-day voluntary testing period where they faced a daily decision to either drop out or continue taking part. Participants were paid for each session they completed.

We saw no evidence for an effect of gamification on attrition, with participants dropping out of each variant at equal rates. Our findings raise doubts about the ability of gamification to increase engagement with cognitive testing studies.
\end{abstract}

Keywords: gamification, gamelike, online, stop signal task, attrition, engagement 


\section{Introduction}

The prospect of assessing cognition remotely and longitudinally is attractive to researchers, health practitioners and pharmaceutical companies alike. However, until recently, assessments of cognitive functioning were typically done in a laboratory or clinical setting, making multiple testing sessions expensive and a burden to both researchers and participants. Recently, the use of online platforms for crowd-sourcing participants, such as such as MTurk (www.mturk.com) and Prolific Academic (prolific.ac), combined with the growing number of platforms for delivering remote cognitive assessments, such as Testable (www.testable.org) and Gorilla (gorilla.sc), have given researchers the ability to test large numbers of people within very short time spans (Peer, Brandimarte, Samat, \& Acquisti, 2017; Woods, Velasco, Levitan, Wan, \& Spence, 2015). These platforms also allow researchers and health professionals to run psychological experiments frequently and inexpensively. However, despite evidence that data collected online is comparable to that collected in a laboratory setting (Crump, McDonnell, \& Gureckis, 2013; Lumsden, Skinner, Woods, Lawrence, \& Munafò, 2016; Schreiner, Reiss, \& Schweizer, 2014; Woods et al., 2015), there are concerns that unsupervised testing environments may result in poorer participant engagement and boredom, leading to poorer quality data, high attrition rates, and rendering such studies unworkable (Eysenbach, 2005).

Gamification may offer a solution to poor participant engagement (Lumsden, Edwards, Lawrence, Coyle, \& Munafò, 2016). The premise is that adding gamelike features to an otherwise mundane task might make it more enjoyable and compelling for the user (Deterding, Sicart, Nacke, O’Hara, \& Dixon, 2011). Indeed, video games are hugely popular ('State of Online Gaming Report', 2013) and deeply engrossing (Rigby \& Ryan, 2011), and by utilising games' ability to engage individuals, we might be able to improve participant performance on the task and reduce the effects of boredom. In previous studies, self-report questionnaires of participant enjoyment have found that gamelike experiments are typically rated as more enjoyable than non-gamelike counterparts (Hawkins, Rae, Nesbitt, \& Brown, 2013; Lumsden, Edwards, et al., 2016; McPherson \& Burns, 2007; Miranda \& Palmer, 2014; Prins, Dovis, Ponsioen, ten Brink, \& van der Oord, 2011; Tong \& Chignell, 
2014). However, despite positive initial findings (Dörrenbächer, Müller, Tröger, \& Kray, 2014; Prins et al., 2011), there is currently little evidence that gamelike tasks can actually make the testing experience sufficiently enjoyable to increase participation. Furthermore, gamification of psychological tasks must be implemented carefully to avoid creating additional cognitive load and affecting the cognitive constructs under test, thus invalidating the task. Although some studies have reported a positive effect of game mechanics on participant performance (Delisle \& Braun, 2011; Dovis, Oord, Wiers, \& Prins, 2011; Ninaus et al., 2015), others have found evidence that gamelike tests do not improve performance, and may in fact worsen it (Hawkins et al., 2013; Katz, Jaeggi, Buschkuehl, Stegman, \& Shah, 2014; Lumsden, Skinner, et al., 2016; McPherson \& Burns, 2007, 2008; Miranda \& Palmer, 2014). For example, (Katz et al., 2014) found that adding a point-scoring system to a working-memory training task negatively impacted the task's ability to train cognition. These contrasted findings are likely due to the diverse range of cognitive tasks being used, and the variety of gamification approaches applied to them, hence highlighting the need for research which systematically manipulates gamification approaches within a single type of task (Lumsden, Edwards, et al., 2016).

We recently conducted a study exploring the impact of two simple game mechanics (Points and Theme) on the data collected by, and subjective participant ratings of, a response inhibition task (Lumsden, Skinner, et al., 2016). The Points variant rewarded participants with points in accordance with their performance on the task while the Theme variant utilised a variety of narratively themed stimuli and task backgrounds. A Non-Game variant was included as a control condition. This was comparable to a clinical version of the task, with some minor graphical changes to ensure suitability for online use. We found that points were rated highest of the three variants on a subjective questionnaire of enjoyment and engagement, and did not negatively affect participant performance on the test. However we found that the narratively themed task was less liked and negatively affected participant performance. In the present study, we aimed to investigate whether simple gamification could reduce participant attrition from an online longitudinal cognitive testing study. We used three variants of another response inhibition task, the Stop Signal Task (SST), with the same gamelike 
features (Non-Game, Points and Theme). To assess the effect of gamification on attrition, we used a longitudinal design whereby participants signed-up to four compulsory test sessions over four consecutive days before entering a six-day voluntary period where they could continue to take part once per day if they desired.

\section{Methods}

\section{Design and Overview}

The aim of this study was to investigate whether three different versions of a SST (NonGame, Points, Theme) would affect participant attrition from a longitudinal cognitive assessment study. We used a between-subjects, repeated measures experimental design that took place online over four to ten days. The dependent variables of interest were the total number of sessions completed each day, Stop Signal Reaction Times (SSRTs), scores on a questionnaire of enjoyment and engagement, and two pilot objective measures of engagement. We pre-registered the study on the Open Science Framework (https://osf.io/ysaqe/).

\section{Participants and Procedure}

Participants were recruited from the user base of Prolific Academic (prolific.ac), which handles the process of checking inclusion criteria, displaying study information and participant reimbursement. We required participants to be older than 18 and to have English as a first language, but had no further exclusion criteria. Once registered, participants were directed to the 'Mindgames Platform' where they entered their Prolific ID and received a unique link, which they used to access the study thereafter. They were then randomly assigned to a single task variant for the duration of the study and completed an online consent form before testing commenced.

Participants were required to complete one session per day for the first four days of the study in order to receive $£ 4$ as compensation for their time. To this end, they were sent daily test reminders via the Prolific Academic messaging system. After four days participants were informed there would be no more reminders, and that they were free to either drop out, or continue to take part in the study 
each day thereafter for up to six days, with each additional session earning them 50p, for a total of between $£ 4$ and $£ 7$.

Ethical approval was obtained from the Faculty of Science Research Ethics Committee at the University of Bristol (40361). The study was conducted according to the revised Declaration of Helsinki ('WMA Declaration of Helsinki - Ethical Principles for Medical Research Involving Human Subjects', 2013). 


\section{Materials}

The Mindgames platform. Aside from participant recruitment, daily reminders and reimbursement, all other elements of the study were hosted on a custom website (mindgames.firebaseapp.com). The website was a single page web app written in JavaScript, with a JSON based Firebase database (firebase.google.com) and PixiJS (www.pixijs.com) as the 2D renderer. The site opened to a main-menu screen from which the participant could view the number of sessions they had completed and the amount of money they'd earned so far (see Figure 1). Participants had access to a 'history' screen which allowed them to view their previous progress and monitor their results over time. Clicking the start button displayed a series of instructions screens followed by the SST task and a short questionnaire. The session ended on the history screen, and the main menu's 'start' button became inactive until midnight that night. Each session took approximately 10 minutes to complete. On the first day of taking part, participants also completed a short demographic questionnaire which collected data on sex, age, ethnicity, level of education and the number of hours spent playing video games each week. See Supplementary Methods for more details of participant demographics.

Stop signal task: Non-Game variant. The SST measures response inhibition, a key feature of executive control (Verbruggen \& Logan, 2008). It tests the participant's 'action restraint' by presenting a series of stimuli to which the participant must respond as quickly as possible, but are occasionally required to withhold a response (Schachar et al., 2007). These 'stop trials' are indicated by a visual warning presented a brief delay after stimulus presentation. The primary outcome measure of the SST is the SSRT which is the number of milliseconds of warning a participant needs for them to be able to successfully inhibit their planned response. In this study we decided to use the SST, as opposed to the Go-NoGo task from our previous study (Lumsden, Skinner, et al., 2016), because the SSRT is theoretically reaction time independent, and we were concerned that latency in the Javascript webapp used to deliver the task might result in artificially inflated reaction times. By using the SST, our primary outcome measure should not be drastically effected even if participant reaction times are longer than anticipated. 
In our SST, each trial began with a fixation cross displayed in the middle of the screen and two coloured zones on the left and right of the fixation cross (see Figure 2A). $500 \mathrm{~ms}$ later a coloured circle appeared over the fixation cross and participants had to respond as rapidly as possible by pressing either the left or right arrow key to indicate which coloured zone matched the colour of the circle. On $25 \%$ of trials, white brackets appeared around the circle after it was shown: when this occurred the subject had to withhold their response and wait until the next trial began (each trial was displayed for $900 \mathrm{~ms}$ ). If the participant responded before the stop signal was displayed, then the trial was recorded as failed, but white brackets were not displayed. Between each trial there was a random inter-trial interval (ITI) of between 500-1000 ms. The delay between the circle onset and the bracket onset is called the Stop Signal Delay (SSD), and was varied according to a four-staircase tracking algorithm, designed to sample evenly across the SSD/Inhibition-Probability space (see Supplementary Methods for full details). The task consisted of five blocks of 48 trials each, with a 10 second break between each block.

In the Non-Game variant, the participant's history was presented as a list of previous sessions, with median reaction times and estimated SSRTs (see Figure 2B). There was also a brief explanation of optimal performance (e.g. "Try to keep both reaction time and SSRT as short as possible"). 
Points variant. The Points variant was similar to the Non-Game variant but with the addition of a points mechanic and the task being framed as a game. Points are a common feature of gamified tasks (Lumsden, Edwards, et al., 2016), and are classed as "1 1 st Step" gamification (Boendermaker, Prins, \& Wiers, 2015). In our task, the participant's points score was displayed at the bottom of the screen throughout (see Figure 2C). The scoring system was very similar to that used in our previous study (Lumsden, Skinner, et al., 2016), which in turn was based on that used by (Miranda \& Palmer, 2014). The scoring system also incorporates findings of (Guitart-Masip et al., 2012) who found that subjects were much more successful in learning active (go) choices when rewarded for them, and passive choices (stop) when punished. On each successful non-stop trial the participant earned points equal to Bonus*(800-RT)/5, and the number of points gained was displayed briefly in the ITI. This Bonus was a multiplier $(\mathrm{x} 2, \mathrm{x} 3, \mathrm{x} 4 \ldots)$, which increased by 1 every 3 trials but decreased by 3 when the participant failed a stop trial. The bonus was not lost on stop trials to which the participant responded before the stop signal was displayed (to all appearances, the trial was not a stop trial). On a successful inhibition to a stop signal, the bonus was not lost, but no points were awarded. Scores were maintained over blocks, but not over sessions. The scoring system was outlined to the participants in the instructions for the task.

The participant's history was presented as a list of median reaction times, SSRTs and scores from each testing session (see Figure 2D). Additionally, the participant's highest score was saved as a High Score, and was displayed in the top right-hand corner throughout every testing session. 
Theme variant. The Theme variant was similar to the Non-Game variant but with the addition of a graphical theme and a sense of progression. The task was framed as a game and featured themed graphics and stimuli, with the yellow and blue stimuli being replaced by images of objects, though still predominantly blue or yellow (see Figure 2E). The task was presented on a series of different graphical backgrounds (see Supplementary Figure 1), but with some shared elements: a conveyor belt on which objects appeared and two bins to the left and right into which these objects were sorted. The stop signal was explained as an automatic "fault detector" which scanned objects as they sat on the conveyor.

The participant's history was presented as a map (see Figure 2F), and previous sessions' summary data was displayed when the user hovered over the corresponding icon. Each level on the map had a unique name and thematic instruction text, with the intention of creating an overarching goal, perceptual curiosity and create a sense of participant progression (Malone, 1980, 1981; Schell, 2008).

Enjoyment and Engagement Questionnaire. The Enjoyment and Engagement Questionnaire was designed to collect subjective ratings of the task and was delivered after every session for all three variants. Session 1,4,7 and 10 delivered the full ten-item questionnaire while the remaining sessions delivered a shorter five item questionnaire. These items were answered using a continuous visual-analogue scale (VAS), presented as a horizontal line 500 pixels long, with a label at either end, and no subdivisions. Participants marked a point between these two labels using their mouse.

The following questions were based on those used in previous studies (Hawkins et al., 2013; Lumsden, Skinner, et al., 2016; Miranda \& Palmer, 2014), and were presented in a random order: (1) How enjoyable did you find that? (2) How frustrating did you find that? (3) How difficult was it to concentrate for the duration of that? (4) How well do you think you performed on that? (L) How mentally stimulating did you find that to be? (6) How boring did you find that? (7) How much effort did you put in throughout that? (8) How repetitive did you find that? (9) How willing would you be to do that again tomorrow? (10) How willing would you be to recommend the study to a friend? 
Questions 2,3,6 and 8 were reverse-scored when calculating a total score, and questions 3,4,6,7 and 10 appeared only in on the long version of the questionnaire.

Objective Measures of Engagement. We piloted two measures that could potentially serve as objective proxies for engagement: we logged the number of times that participants hid the browser window (lost focus) while completing the SST, hypothesising that unengaged participants would be more likely to briefly visit other websites while testing. We also investigated coefficients of variation, which quantify reaction time intra-individual variability with respect to mean reaction time, as there is some evidence that changes in motivation can be reflected in reaction time variation (Andreou et al., 2007).

\section{Statistical Analysis}

Since the three task variants were designed with the aim of minimising differences in cognitive data, and Frequentist statistics is not ideal for testing equivalences (Berger \& Sellke, 1987; Blackwelder, 1982), we used Bayesian approaches to assess whether comparable means could be considered equivalent (Wetzels, Raaijmakers, Jakab, \& Wagenmakers, 2009). Where Frequentist approaches found no evidence of a difference between two means, we used Bayesian t-tests to assess the evidence for equality (Rouder, Speckman, Sun, Morey, \& Iverson, 2009). A Bayesian t-test produces a Bayes Factor, which either provides evidence to support one of two hypotheses, or implies the data are insensitive (see Table 1). In our analysis one hypothesis was always "the mean difference is zero" and the other was "the mean difference is not zero." We used the Bayesian t-test procedure JASP, with a Cauchy prior width of 0.707 .

For brevity, not all planned analyses are presented below - for more detailed methods and analyses please see the Supplementary Information. 
Sample Size Determination. To the best of our knowledge, no other studies have investigated the impact of gamification on attrition rates from a cognitive testing programme and therefore we had no previous effect sizes on which to base a sample size determination. However, we performed a power analysis to calculate the sample size required to detect a difference of $13 \%$ in dropout between task variants using the Kaplan-Meier/Log-rank method. With alpha $=0.05$ and $80 \%$ power we required $\mathrm{n}=291$.

Variable calculation. The main variables of interest were the total number of sessions completed each day, subjective ratings, objective measures of engagement and SSRTs. Estimated SSRTs were calculated automatically at the end of each session using the integration method as detailed in (Band, van der Molen, \& Logan, 2003; Logan, 1994). However, given our staircase algorithm sampled evenly across inhibition space, the optimal method for calculating SSRTs was to fit an inhibition function for each session using a probit curve model, and then calculate a summary SSRT using the SSD where P(Respond|Signal $)=0.5$, as detailed in (Logan, 1994; Logan, Schachar, $\&$ Tannock, 1997). We used boxplots and a one-way ANOVA with task variant as the betweensubjects factor to investigate the effects of gamification on SSRT. We combined data from the first four sessions, and

Differences in attrition curves were assessed visually using the Kaplan Meier method, a LogRank test, and a one-way ANOVA of 'number of sessions completed'. Again, we used post-hoc ttests to explore differences between groups, and where we found no evidence for a difference we used Bayesian t-tests to assess the evidence for equality.

We assessed differences in subjective ratings both visually, using bar-charts, and using a repeated-measures ANOVA of total score with session number as the time factor and task variant as the between-subjects factor. We used one-way ANOVAs with data combined across the first four sessions to investigate differences in coefficient of variation and loss of focus events between task variants. 


\section{Results}

\section{Characteristics of Participants}

A total of 482 participants signed up to take part in this study, with $419(87 \%)$ of those completing at least one session. A total of 265 (55\%) participants completed four sessions over four consecutive days as was required by the study criteria: henceforth called conforming participants. We excluded five participants from the main analysis because their reaction times or non-stop accuracy scores were more than four interquartile ranges away from their group median. We excluded data from sessions that were started but not completed, and we removed trials from the analysis where participants responded in less than $150 \mathrm{~ms}$.

The analysis below presents data from 260 participants: less than our intended sample size of 291. This because 32 participants failed to complete the required four sessions in four days, but instead managed to complete four sessions within five days. During the study, we intended on including these loosely conforming participants in the analysis, and so stopped recruitment once our intended sample size was achieved. However, for simplicity and adherence to the protocol, we have now decided to present only strictly conforming participant's data below. Analysis of non-conforming and loosely conforming participants' attrition is presented in Supplementary Analyses.

Excluding outliers, 260 conforming participants took part: 91 in the Non-Game variant (mean age $=36, \mathrm{SD}=17,47 \%$ male), 86 in the Points variant (mean age $=35, \mathrm{SD}=12,57 \%$ male), and 83 in the Theme (mean age $=34, \mathrm{SD}=11,51 \%$ male). A chi-square test showed no evidence for a difference in the number of male participants in each group $\left(X^{2}(2, N=260)=1.717, p=0.42\right)$ and a one-way ANOVA of participant age showed no evidence of differences between task variants $(F$ $[2,257]=0.698, p=0.50$, partial $\left.\eta^{2}=0.005\right)$. The number of hours spent playing video games was comparable between the groups: Non-Game (mean $=5, \mathrm{SD}=4)$, Points (mean $=8, \mathrm{SD}=16)$ and Theme (mean $=8, \mathrm{SD}=14$ ), with a one-way ANOVA showing no evidence of differences between task variants $\left(F[2,257]=0.345, p=0.71\right.$, partial $\left.\eta^{2}=0.003\right)$. Participants had a high level of education (median = "Bachelor's Degree" in all task variants), again with a one-way ANOVA showing no evidence of differences between task variants $\left(F[2,257]=2.339, p=0.098\right.$, partial $\eta^{2}=$ 
0.018). The majority were of European ancestry $(88 \%)$ with a chi-square test indicating no clear evidence of different distributions of ethnicities between the three variants $\left(X^{2}(12, N=260)=16.16\right.$, $p=0.18)$. The most common browser used to complete the experiment was Google Chrome (71\%), with others including Firefox (19\%), Netscape (5\%), Safari (4\%), Opera (0.5\%) and Internet Explorer $(0.5 \%)$.

\section{Attrition}

Figure 3 shows the attrition of conforming participants, while Table 2 shows the mean number of sessions completed by participants in each variant. We used the Kaplan Meier method to calculate estimated survival times. A Log-Rank test showed no evidence of a difference between the distributions $\left(X^{2}(2, N=265)=3.022, p=0.22\right)$ and a one-way ANOVA of the number of sessions completed also found no clear evidence of a difference between task variants $(F[2,262]=1.534, p=$ 0.21 , partial $\eta^{2}=0.012$ ). Given the similarity between Non-Game and Points in mean number of sessions completed, we used a Bayesian T-test to assess their equality and found substantial evidence that they were equal $($ Bayes factor $=0.163)$, but there was no evidence of equality between the Theme and the Points variant $(\mathrm{BF}=0.493)$ or the Non-Game variant $(\mathrm{BF}=0.483)$.

\section{Measures of Subjective Engagement}

We used a repeated-measures ANOVA of mean score from the Enjoyment and Engagement questionnaire with session number $(1,4)$ as the within-subjects factor, and task variant as the between. We used only the two full-length questionnaires completed on the $1^{\text {st }}$ and the $4^{\text {th }}$ session, and completed by all participants (for short-form questionnaire results see Supplementary Analyses). We saw evidence for small effects of both task variant $\left(F[2,261]=3.805, p=0.024\right.$, partial $\left.\eta^{2}=0.028\right)$ and time $\left(F[1,261]=35.693, p<0.001\right.$, partial $\left.\eta^{2}=0.120\right)$, and weak evidence of an interaction $(F$ $[2,261]=3.014, p=0.051$, partial $\left.\eta^{2}=0.023\right)$. We saw ratings of all task variants decrease between the first ( $M=56,95 \%$ CI 54 to 57) and fourth session ( $M=51,95 \%$ CI 49 to 53), but it appears the Non-Game and Points variants were the main drivers of the interaction effect: dropping by $6 \%$ (95\% CI 4 to 8 ) between Session 1 and Session 4, whereas ratings of the Theme task decreased only by $2 \%$ 
(95\% CI -1 to 5). Figure 4 shows the mean scores from each task variant at the two timepoints, and a breakdown of ratings by individual questions is presented in the Supplementary Analyses.

As an unplanned exploratory analysis, we were interested in whether a participant's rating one day predicted their return to the study on the following day. We ran a logistic regression with "returned following day" as the binary dependant variable and the previous day's score on the subjective questionnaire as the predictor variable. However, we saw no evidence that subjective questionnaire scores predicted return the following day, $(\beta=0.008, \mathrm{SE}=0.005$, Wald $(1)=2.166, p=$ $0.141, \mathrm{OR}=1.001,95 \%$ CI 0.997 to 1.019$)$

\section{Stop signal reaction times.}

We calculated SSRTs for each session separately, excluding sessions where the assumptions of the race model did not hold, meaning that any calculated SSRTs would not be good representations of the data (see (Logan, 1994). To that end, we excluded sessions where the median non-stop trial reaction time was longer than the median failed-stop trial reaction time, where SSDs were not positively correlated with their corresponding median failed-stop reaction times, and where stop-trial accuracy was not negative correlated with SSD. Of the 1050 sessions assessed, we excluded 161: 75 from the Non-Game variant, 37 from Points and 49 from Theme. 3 participants failed to meet the assumptions of the race model in all four compulsory sessions, resulting in their exclusion from this analysis. We then analysed each participant's mean SSRT from the first four sessions, with boxplots shown in Figure 5.

A one-way ANOVA showed weak evidence of a small effect of task variant on SSRT ( $F$ $[2,255]=2.954, p=0.054$, partial $\eta^{2}=0.022$ ) with post-hoc t-tests showing a difference between the Theme variant $(M=289, S D=67)$ and Points variant $(M=266, S D=66)$ (mean difference $=23$, $95 \%$ CI 5 to $42, \mathrm{t}(169)=2.386, p=0.047, d=0.35)$. There was no evidence for other differences $(p \mathrm{~s}$ $>0.24$ ). Bayesian t-tests showed no evidence of equality between the SSRTs of the Non-Game and Theme variants $(\mathrm{BF}=0.590)$, but found substantial evidence for equality between the Non-Game $(\mathrm{M}$ $=274, \mathrm{SD}=55)$ and the Points variants $(\mathrm{BF}=0.223)$. 


\section{Measures of Objective Engagement}

To see whether differences in subjective ratings could be corroborated by objective measures of engagement, we analysed reaction time intra-individual variability and website loss of focus events from the four compulsory sessions combined (see Table 3). A one-way ANOVA of coefficient of variation showed strong evidence for a medium effect of task variant $(F[2,260]=6.985, p=0.001$, partial $\left.\eta^{2}=0.051\right)$ on participants' reaction time variability, with lower coefficients indicating there was less variability. Post-hoc t-tests showed strong evidence of a difference between the Points and Theme variants (mean difference $=0.67,95 \%$ CI 0.32 to $1.02, \mathrm{t}(174)=3.766, p<0.001, d=0.57$ ), but no clear evidence for other differences were found ( $p s>0.06)$.

Loss-of-Focus events were rare in all task variants, with each participant switching away from the less than once per session on average. Regardless, we assessed differences in Loss-Of-Focus Events between the three task variants using a one-way ANOVA but found no evidence for any differences $\left(F[2,260]=1.137, p=0.32\right.$, partial $\left.\eta^{2}=0.008\right)$.

\section{Discussion}

Overall, we saw no clear evidence of an effect of task variant on participant attrition. This null finding is further strengthened when we include data from loosely conforming participants (see Supplementary Analyses), which show strong evidence that the mean number of sessions completed was equal in all task variants. To the best of our knowledge, this is the first empirical study examining the effects of gamification on participant adherence to a testing regime, and our results raise doubts about the efficacy of gamelike tasks for increasing participant engagement.

Despite there being no difference in usage between the variants, we did see differences in the subjective ratings of the tasks. One possible explanation for these findings relates to Selfdetermination theory; a popular theory of motivation which centres around the concept of psychological needs and need-satisfaction. Self-determination theory posits that human beings have three needs: competence, autonomy and relatedness, and that we find activities to be intrinsically 
motivating if they help us to fulfil these needs (Deci \& Ryan, 2002). In the case of our gamelike variants, the Points variant would seem to address competency needs by providing constant feedback which reinforces the player's success, but we do not consider the Theme variant or the Non-Game variant to adequately meet any of the three needs. Since the Points variant was the only variant to address any of these needs, this may explain why it was rated as the most enjoyable in both this study and our previous study (Lumsden, Skinner, et al., 2016).

The Theme variant was rated as the worst of the three tasks, which was surprising as it maintained the highest percentage of participants until day ten. One potential explanation is that the task was framed as a game and looked like a game, but offered no actual gameplay. Secondly, the map screen and changing graphical backgrounds may have hinted at player autonomy and exploration as is typical in other games, but ultimately the player experience was railroaded. These two factors may have undermined autonomy and violated participant expectations, resulting in a dissatisfying experience (Boendermaker, Maceiras, Boffo, \& Wiers, 2016; Ryan, Rigby, \& Przybylski, 2006). Despite this, it is possible that the clear end goal on the map and novelty of changing backgrounds could explain the maintenance of participants in the Theme variant, while still not being a very satisfying or enjoyable experience.

One additional factor to consider, in the light of self-determination theory, is that paying participants in attrition studies such as this may be counterproductive to measuring true engagement. There is evidence that providing extrinsic rewards for otherwise motivating tasks may undermine participant autonomy, therefore affecting the task's ability to meet our psychological needs (Deci, Ryan, \& Koestner, 1999; Deterding, 2011). In this study, it is impossible to determine whether intrinsic motivation to take part was affected by the incentive of 50p per additional session, and it would therefore be prudent to run a similar attrition study but without incentives. Money can be a powerful motivator; for example, (Khadjesari et al., 2011) showed found that offering a $£ 10$ Amazon voucher to each participant in a longitudinal study resulted in a $9 \%$ increased response rate. In our case, it may simply be that money was the most important factor for taking part, and that the similar attrition rates were driven by the identical incentives. Given that the development costs of gamelike- 
tasks can be substantial, and that such tasks often require several stages of validation before use, it likely remains the case that the most cost-effective method of reducing attrition is simply to offer unconditional monetary rewards.

We also found no evidence that participant ratings of engagement and enjoyment could predict the number of optional sessions they would complete. This, combined with the disconnect between the Theme variant ratings and Theme variant usage, serves to highlight the split in different types of engagement that has recently begun to be conceptualised in the literature (Perski, Blandford, West, \& Michie, 2016). In short, the word engagement has been used in the past to refer to both engagement as subjective experience and engagement as usage, and this study is further evidence that the two concepts are not as closely related as one might assume. Evidence from the video game literature has found that game enjoyment does not relate strongly to game usage, and that game usage can be driven by many other factors including boredom, loneliness and need for escapism (Boyle, Connolly, Hainey, \& Boyle, 2012; Lee \& LaRose, 2007). This highlights the need for future studies of engagement to measure both user-reported engagement and objective

Our two pilot objective measures of engagement (reaction time variation and loss-of-focus events) show a pattern in line with subjective ratings. That is, the Points variant had the least variable reaction times, the fewest loss-of-focus events and was rated the highest. The Non-Game variant was similar though slightly worse, and the Theme variant showed more variability, more losses of focus and lower subjective ratings than either. Given that the subjective questionnaire measures were collected immediately after the task was delivered, these corresponding patterns serve as a positive indicator that these objective measures are related to engagement as subjective experience.

When assessing cognitive data, we found small detrimental effects of game mechanics on almost all measures (see Supplementary Information). In the case of SSRTs however, we found evidence of equality between the Points variant and the Non-Game variant. Though the Points variant had longer non-stop reaction times, it is possible that the highly salient feedback and motivational effect of points served to increase participant performance, as has been found in a number of previous studies (Attali \& Arieli-Attali, 2015; Leotti \& Wager, 2010; Ninaus et al., 2015). 


\section{Limitations and Conclusions}

We consider the fact that we did not meet our intended sample size an important limitation of this study. However, we maintain that the results of our supplementary analyses including the loosely conforming participants are quite conclusive, and strengthen our finding that there was no effect of gamification on attrition. Nevertheless, we accept that a balanced group analysis would be preferable. Secondly, we acknowledge that our sample, recruited from Prolific Academic, with high levels of education, may not be representative of the wider population. Thirdly, we acknowledge that the design of study used is not suitable to validate our gamelike variants as measures of response inhibition, as that would require a within-subjects design in order to test predictive validity (Boendermaker et al., 2015; Kato, 2013). Fourthly, the gamelike features we implemented were very lightweight, and certainly wouldn't constitute a full game. Indeed, neither of our games were likely enjoyable enough that a participant would consider doing them for their own sake. Though this was a necessary to try to reduce the impact of gamification on the cognitive data, it likely reduced any effects of gamification we might've seen. Fifthly, the time course of our study, which took place over days, may not be informative about attrition in studies that take place over longer periods of weeks or months. Finally, as mentioned previously, there are issues relating to motivation and incentives, as in reality participants completing cognitive assessments will be presented with requests to complete a study over a fixed period for a fixed fee, and not with the option to continue for additional recompense.

In conclusion, the Theme variant had negative effects on the cognitive data and showed no clear evidence of reducing attrition. It was also rated as the least enjoyable and was the task switched away from most often. This suggests that themed gamelike tasks, at least those that use graphics alone, are non-optimal for use in cognitive assessment studies. In contrast, and replicating our previous finding (Lumsden, Skinner, et al., 2016), subjective ratings showed the Points variant to be well received. We saw detrimental effects on reaction times and inhibition accuracy, but the calculation process of SSRTs accounts for both these measures somewhat, and indeed we found 
SSRTs from the Points and Non-game variants to be equal. This shows that points can an effective way of increasing participant enjoyment of a cognitive task while still collecting valid data.

Despite differences in subjective ratings between the task variants, we saw no effect of gamification on participant attrition over the six-day optional testing period. Gamification has been promoted as a potential solution to engagement problems in both psychology and digital health care for several years, but we found no effect of gamification on engagement as usage in this case. The term gamification may have existed for a decade, but the formalisation of gamification's implementation and effectiveness is only just beginning, and there is clearly further work to be done to understand how we can translate differences in subjective ratings to differences in usage. 


\section{Acknowledgements}

The authors gratefully acknowledge the artistic contribution of Melissa Groves who provided graphical resources for the themed task variant. The authors are members of the United Kingdom Centre for Tobacco and Alcohol Studies, a UKCRC Public Health Research: Centre of Excellence. Funding from British Heart Foundation, Cancer Research UK, Economic and Social Research Council, Medical Research Council, and the National Institute for Health Research, under the auspices of the UK Clinical Research Collaboration, is gratefully acknowledged. The UK Medical Research Council and the Wellcome Trust (092731) and the University of Bristol provide core support for ALSPAC. This work was supported by the Medical Research Council (MC_UU_12013/6 and MC_UU_12013/7), a PhD studentship to JL funded by the Economic and Social Research Council and Cambridge Cognition Limited. The funders had no role in review design, data extraction and analysis, decision to publish, or preparation of the manuscript. JL and MM designed the study. JL programmed the task software and collected the data. JL analysed the data. AS, NL and MM contributed to development of the manuscript. 


\section{Conflicts of Interest}

None declared 


\section{References}

Andreou, P., Neale, B. M., Chen, W., Christiansen, H., Gabriels, I., Heise, A., ... Kuntsi, J. (2007). Reaction time performance in ADHD: improvement under fast-incentive condition and familial effects. Psychological Medicine, 37(12), 1703-1715. https://doi.org/10.1017/S0033291707000815

Attali, Y., \& Arieli-Attali, M. (2015). Gamification in assessment: Do points affect test performance? Computers \& Education, 83, 57-63. https://doi.org/10.1016/j.compedu.2014.12.012

Band, G. P. H., van der Molen, M. W., \& Logan, G. D. (2003). Horse-race model simulations of the stop-signal procedure. Acta Psychologica, 112(2), 105-142. https://doi.org/10.1016/S00016918(02)00079-3

Berger, J. O., \& Sellke, T. (1987). Testing a Point Null Hypothesis: The Irreconcilability of P Values and Evidence. Journal of the American Statistical Association, 82(397), 112-122. https://doi.org/10.1080/01621459.1987.10478397

Blackwelder, W. C. (1982). 'Proving the null hypothesis' in clinical trials. Controlled Clinical Trials, 3(4), 345-353. https://doi.org/10.1016/0197-2456(82)90024-1

Boendermaker, W. J., Maceiras, S. S., Boffo, M., \& Wiers, R. W. (2016). Attentional Bias Modification With Serious Game Elements: Evaluating the Shots Game. JMIR Serious Games, 4(2), e20. https://doi.org/10.2196/games.6464

Boendermaker, W. J., Prins, P. J. M., \& Wiers, R. W. (2015). Cognitive Bias Modification for adolescents with substance use problems - Can serious games help? Journal of Behavior Therapy and Experimental Psychiatry, 49, Part A, 13-20. https://doi.org/10.1016/j.jbtep.2015.03.008

Boyle, E. A., Connolly, T. M., Hainey, T., \& Boyle, J. M. (2012). Engagement in digital entertainment games: A systematic review. Computers in Human Behavior, 28(3), 771-780. https://doi.org/10.1016/j.chb.2011.11.020 
Crump, M. J. C., McDonnell, J. V., \& Gureckis, T. M. (2013). Evaluating Amazon's Mechanical Turk as a Tool for Experimental Behavioral Research. PLoS ONE, 8(3), e57410. https://doi.org/10.1371/journal.pone.0057410

Deci, E. L., Ryan, R. M., \& Koestner, R. (1999). A meta-analytic review of experiments examining the effects of extrinsic rewards on intrinsic motivation. Psychological Bulletin, 125(6), 627668.

Deci, \& Ryan, R. M. (2002). Handbook of self-determination research. University Rochester Press.

Delisle, J., \& Braun, C. M. J. (2011). A Context for Normalizing Impulsiveness at Work for Adults with Attention Deficit/Hyperactivity Disorder (Combined Type). Arch. Clin. Neuropsychol., $26,602-613$.

Deterding, S. (2011). Situated motivational affordances of game elements: A conceptual model. In Gamification: Using game design elements in non-gaming contexts, a workshop at CHI.

Deterding, S., Sicart, M., Nacke, L., O’Hara, K., \& Dixon, D. (2011). Gamification. Using Gamedesign Elements in Non-gaming Contexts. In CHI '11 Extended Abstracts on Human Factors in Computing Systems (pp. 2425-2428). New York, NY, USA: ACM. https://doi.org/10.1145/1979742.1979575

Dörrenbächer, S., Müller, P. M., Tröger, J., \& Kray, J. (2014). Dissociable effects of game elements on motivation and cognition in a task-switching training in middle childhood. Cognition, 5, 1275. https://doi.org/10.3389/fpsyg.2014.01275

Dovis, Oord, Wiers, \& Prins. (2011). Can Motivation Normalize Working Memory and Task Persistence in Children with Attention-Deficit/Hyperactivity Disorder? The Effects of Money and Computer-Gaming. Journal of Abnormal Child Psychology, 40(5), 669-681. https://doi.org/10.1007/s10802-011-9601-8

Eysenbach, G. (2005). The Law of Attrition. Journal of Medical Internet Research, 7(1), e11. https://doi.org/10.2196/jmir.7.1.e11 
Guitart-Masip, M., Huys, Q. J. M., Fuentemilla, L., Dayan, P., Duzel, E., \& Dolan, R. J. (2012). Go and no-go learning in reward and punishment: interactions between affect and effect. NeuroImage, 62(1), 154-166. https://doi.org/10.1016/j.neuroimage.2012.04.024

Hawkins, G. E., Rae, B., Nesbitt, K. V., \& Brown, S. D. (2013). Gamelike features might not improve data. Behavior Research Methods, 45(2), 301-318. https://doi.org/10.3758/s13428-012-02643

Kato, P. M. (2013, April 25). What do you mean when you say your serious game has been validated? Experimental vs. Test Validity. Retrieved from http://www.webcitation.org/6gt9POLIu

Katz, B., Jaeggi, S., Buschkuehl, M., Stegman, A., \& Shah, P. (2014). Differential effect of motivational features on training improvements in school-based cognitive training. Frontiers in Human Neuroscience, 8, 242. https://doi.org/10.3389/fnhum.2014.00242

Khadjesari, Z., Murray, E., Kalaitzaki, E., White, I. R., McCambridge, J., Thompson, S. G., ... Godfrey, C. (2011). Impact and Costs of Incentives to Reduce Attrition in Online Trials: Two Randomized Controlled Trials. Journal of Medical Internet Research, 13(1), e26. https://doi.org/10.2196/jmir.1523

Lee, D., \& LaRose, R. (2007). A Socio-Cognitive Model of Video Game Usage. Journal of Broadcasting and Electronic Media, 51, 632-650.

Leotti, L. A., \& Wager, T. D. (2010). Motivational influences on response inhibition measures. Journal of Experimental Psychology. Human Perception and Performance, 36(2), 430-447. https://doi.org/10.1037/a0016802

Logan, G. D. (1994). On the ability to inhibit thought and action: A users' guide to the stop signal paradigm. In D. Dagenbach \& T. H. Carr (Eds.), Inhibitory processes in attention, memory, and language (pp. 189-239). San Diego, CA, US: Academic Press.

Logan, G. D., Schachar, R. J., \& Tannock, R. (1997). Impulsivity and Inhibitory Control. Psychological Science, 8(1), 60-64. https://doi.org/10.1111/j.1467-9280.1997.tb00545.x 
Lumsden, J., Edwards, E. A., Lawrence, N. S., Coyle, D., \& Munafò, M. R. (2016). Gamification of Cognitive Assessment and Cognitive Training: A Systematic Review of Applications and Efficacy. JMIR Serious Games, 4(2), e11. https://doi.org/10.2196/games.5888

Lumsden, J., Skinner, A., Woods, A., Lawrence, N., \& Munafò, M. (2016). The effects of gamelike features and test location on cognitive test performance and participant enjoyment. PeerJ.

Malone, T. W. (1980). What Makes Things Fun to Learn? Heuristics for Designing Instructional Computer Games. In Proceedings of the 3rd ACM SIGSMALL Symposium and the First SIGPC Symposium on Small Systems (pp. 162-169). New York, NY, USA: ACM. https://doi.org/10.1145/800088.802839

Malone, T. W. (1981). Toward a theory of intrinsically motivating instruction. Cognitive Science, 5(4), 333-369. https://doi.org/10.1016/S0364-0213(81)80017-1

McPherson, \& Burns. (2007). Gs Invaders: Assessing a computer game-like test of processing speed. Behavior Research Methods.

McPherson, \& Burns. (2008). Assessing the validity of computer-game-like tests of processing speed and working memory. Behavior Research Methods.

Miranda, A. T., \& Palmer, E. M. (2014). Intrinsic motivation and attentional capture from gamelike features in a visual search task. Behavior Research Methods, 46(1), 159-172. https://doi.org/10.3758/s13428-013-0357-7

Ninaus, M., Pereira, G., Stefitz, R., Prada, R., Paiva, A., Neuper, C., \& Wood, G. (2015). Game elements improve performance in a working memory training task. International Journal of Serious Games, 2(1). https://doi.org/10.17083/ijsg.v2i1.60

Peer, E., Brandimarte, L., Samat, S., \& Acquisti, A. (2017). Beyond the Turk: Alternative platforms for crowdsourcing behavioral research. Journal of Experimental Social Psychology, 70, 153163. https://doi.org/10.1016/j.jesp.2017.01.006

Perski, O., Blandford, A., West, R., \& Michie, S. (2016). Conceptualising engagement with digital behaviour change interventions: a systematic review using principles from critical interpretive 
synthesis. Translational Behavioral Medicine, 1-14. https://doi.org/10.1007/s13142-0160453-1

Prins, P. J. M., Dovis, S., Ponsioen, A., ten Brink, E., \& van der Oord, S. (2011). Does computerized working memory training with game elements enhance motivation and training efficacy in children with ADHD? Cyberpsychology, Behavior and Social Networking, 14(3), 115-122. https://doi.org/10.1089/cyber.2009.0206

Raftery, A. E. (1995). Bayesian Model Selection in Social Research. Sociological Methodology, 25, 111-163. https://doi.org/10.2307/271063

Rigby, S., \& Ryan, R. M. (2011). Glued to games: How video games draw us in and hold us spellbound, 186.

Rouder, J. N., Speckman, P. L., Sun, D., Morey, R. D., \& Iverson, G. (2009). Bayesian t tests for accepting and rejecting the null hypothesis. Psychonomic Bulletin \& Review, 16(2), 225-237. https://doi.org/10.3758/PBR.16.2.225

Ryan, R. M., Rigby, C. S., \& Przybylski, A. (2006). The Motivational Pull of Video Games: A SelfDetermination Theory Approach. Motivation and Emotion, 30(4), 344-360. https://doi.org/10.1007/s11031-006-9051-8

Schachar, R., Logan, G. D., Robaey, P., Chen, S., Ickowicz, A., \& Barr, C. (2007). Restraint and Cancellation: Multiple Inhibition Deficits in Attention Deficit Hyperactivity Disorder. Journal of Abnormal Child Psychology, 35(2), 229-238. https://doi.org/10.1007/s10802-006$9075-2$

Schell, J. (2008). The Art of Game Design. CRC Press. Retrieved from http://www.goodreads.com/work/best_book/3436929-the-art-of-game-design-a-book-oflenses

Schreiner, M., Reiss, S., \& Schweizer, K. (2014). Method Effects on Assessing Equivalence of Online and Offline Administration of a Cognitive Measure: The Exchange Test. International Journal of Internet Science, 9(1), 52-63. 
State of Online Gaming Report. (2013). Spilgames. Retrieved from http://www.webcitation.org/6gt7YwEGt

Tong, T., \& Chignell, M. (2014). Developing a Serious Game for Cognitive Assessment: Choosing Settings and Measuring Performance. In Proceedings of the Second International Symposium of Chinese CHI (pp. 70-79). https://doi.org/10.1145/2592235.2592246

Verbruggen, F., \& Logan, G. D. (2008). Response inhibition in the stop-signal paradigm. Trends in Cognitive Sciences, 12(11), 418-424. https://doi.org/10.1016/j.tics.2008.07.005

Wetzels, R., Raaijmakers, J. G. W., Jakab, E., \& Wagenmakers, E.-J. (2009). How to quantify support for and against the null hypothesis: A flexible WinBUGS implementation of a default Bayesian t test. Psychonomic Bulletin \& Review, 16(4), 752-760. https://doi.org/10.3758/PBR.16.4.752

WMA Declaration of Helsinki - Ethical Principles for Medical Research Involving Human Subjects. (2013, October 19). Retrieved 20 June 2016, from http://www.wma.net/en/30publications/10policies/b3/index.html

Woods, A. T., Velasco, C., Levitan, C. A., Wan, X., \& Spence, C. (2015). Conducting perception research over the internet: a tutorial review. PeerJ, 3, e1058. https://doi.org/10.7717/peerj.1058 


\section{Tables}

Table 1: Interpreting Bayes Factors (adapted from (Raftery, 1995))

\begin{tabular}{|l|l|l|}
\hline $\begin{array}{l}\text { Hypothesis 0: The difference } \\
\text { between means is 0 }\end{array}$ & Strength of evidence & $\begin{array}{l}\text { Hypothesis 1: The difference } \\
\text { between means is between 0 } \\
\text { and X }\end{array}$ \\
\hline $.33 \leq \mathrm{BF} \leq 1$ & No support either way & $1 \leq \mathrm{BF} \leq 3$ \\
\hline $.1 \leq \mathrm{BF} \leq .33$ & Positive & $3 \leq \mathrm{BF} \leq 10$ \\
\hline $.01 \leq \mathrm{BF} \leq .1$ & Strong & $10 \leq \mathrm{BF} \leq 100$ \\
\hline $\mathrm{BF}<.01$ & Decisive & $\mathrm{BF}>100$ \\
\hline
\end{tabular}


Table 2: Mean number of sessions completed, shown separately by task variant.

\begin{tabular}{|l|l|l|}
\hline & $\begin{array}{l}\text { All participants } \\
\text { (95\% CI) }\end{array}$ & $\begin{array}{l}\text { Conforming participants } \\
\text { (95\% CI) }\end{array}$ \\
\hline Non-Game & $4.9(4.4$ to 5.5) & $7.4(6.8$ to 8.0$)$ \\
\hline Points & $5.1(4.5$ to 5.6) & $7.5(7.0$ to 8.0$)$ \\
\hline Theme & $5.3(4.7$ to 5.9) & $8.0(7.5$ to 8.6$)$ \\
\hline
\end{tabular}

Conforming participants are those who completed their first four sessions within four days as required. 'All participants' includes all who signed up, regardless of their number of sessions completed. 
Table 3: Mean objective measures of participant engagement from the first four sessions, shown separately by task variant.

\begin{tabular}{|l|l|l|}
\hline & $\begin{array}{l}\text { Coefficient of variation } \\
\text { (95\% CI) }\end{array}$ & $\begin{array}{l}\text { Loss-of-focus events } \\
\text { (95\% CI) }\end{array}$ \\
\hline Non-Game & $5.93(5.68$ to 6.18$)$ & $0.85(0.50$ to 1.19$)$ \\
\hline Points & 5.67 $(5.43$ to 5.91$)$ & $0.82(0.43$ to 1.20$)$ \\
\hline Theme & $6.34(6.09$ to 5.59$)$ & $1.21(0.75$ to 1.67$)$ \\
\hline Overall & 5.98 (5.83 to 6.12) & $0.95(0.72$ to 1.18$)$ \\
\hline
\end{tabular}




\section{Figures}

Figure 1: Menu screens of the three task variants.
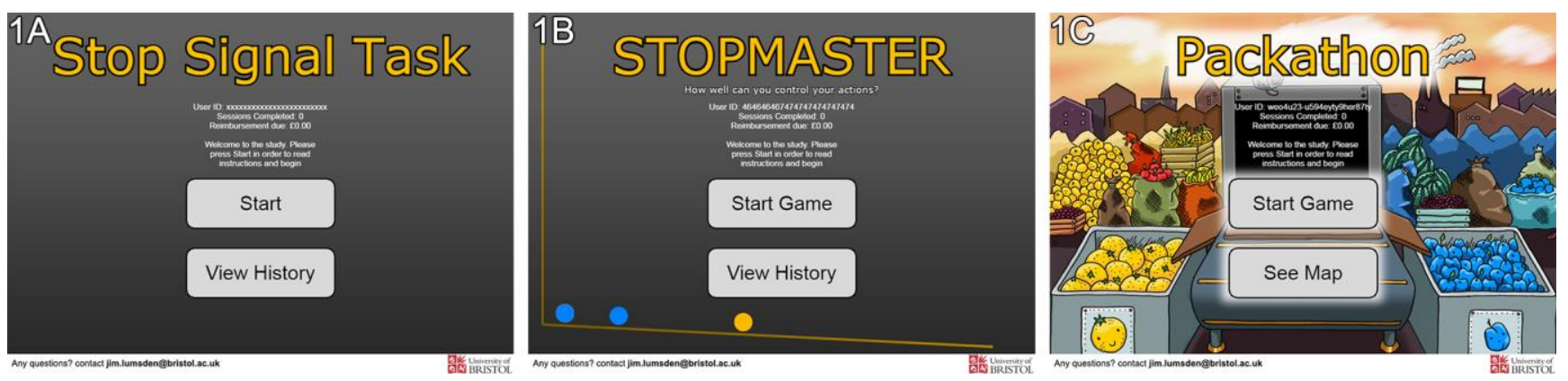

(A) Non-Game variant, (B) Points variant, (C) Theme Variant 
Figure 2: Screenshots of the Stop Signal Task variants and their associated history Screens
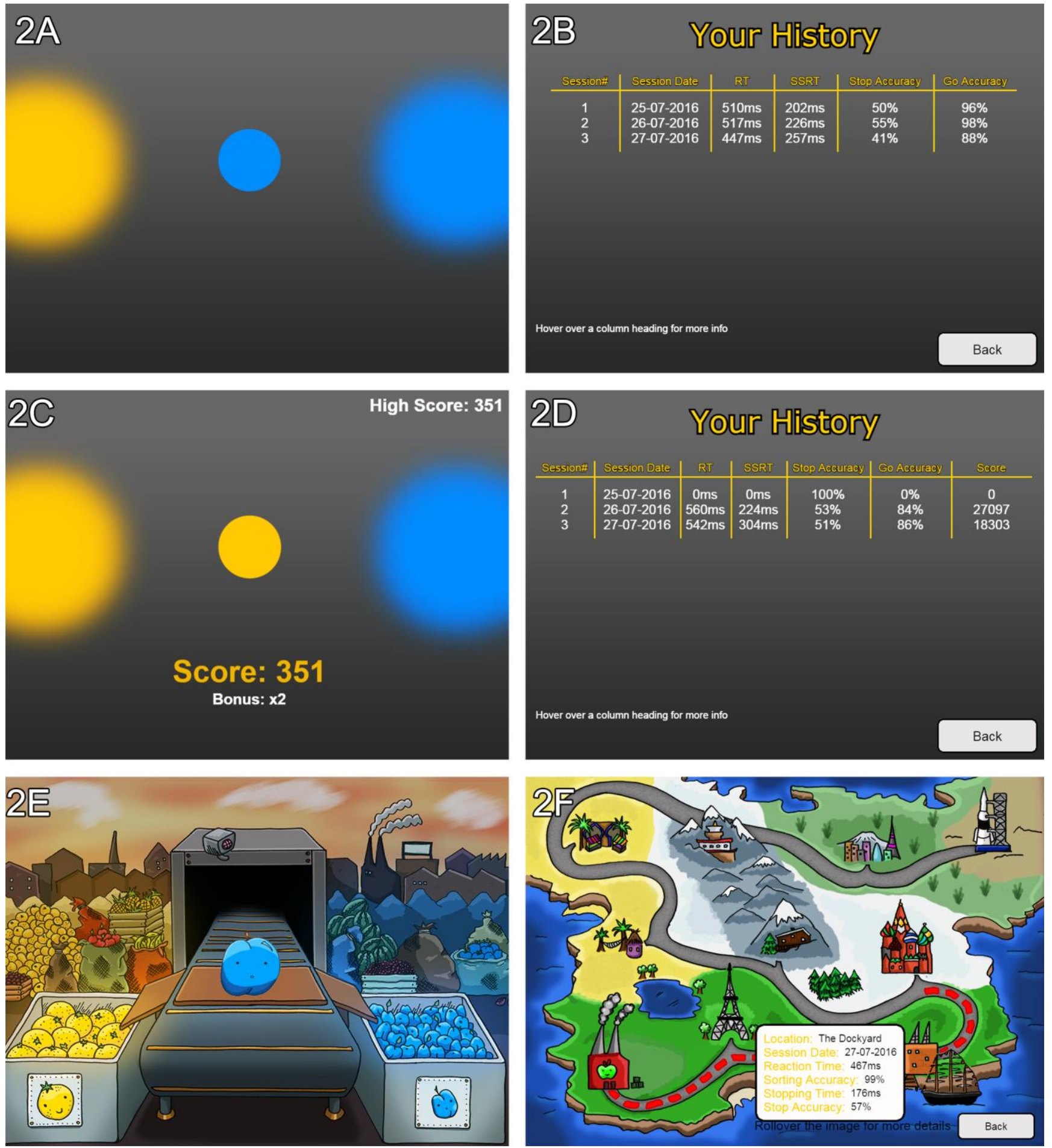

(A/B) Non-Game variant, (C/D) Points variant, (E/F) Theme variant 
Figure 3: Percentage of conforming participants plotted against the number of sessions they completed, shown separately by task variant.

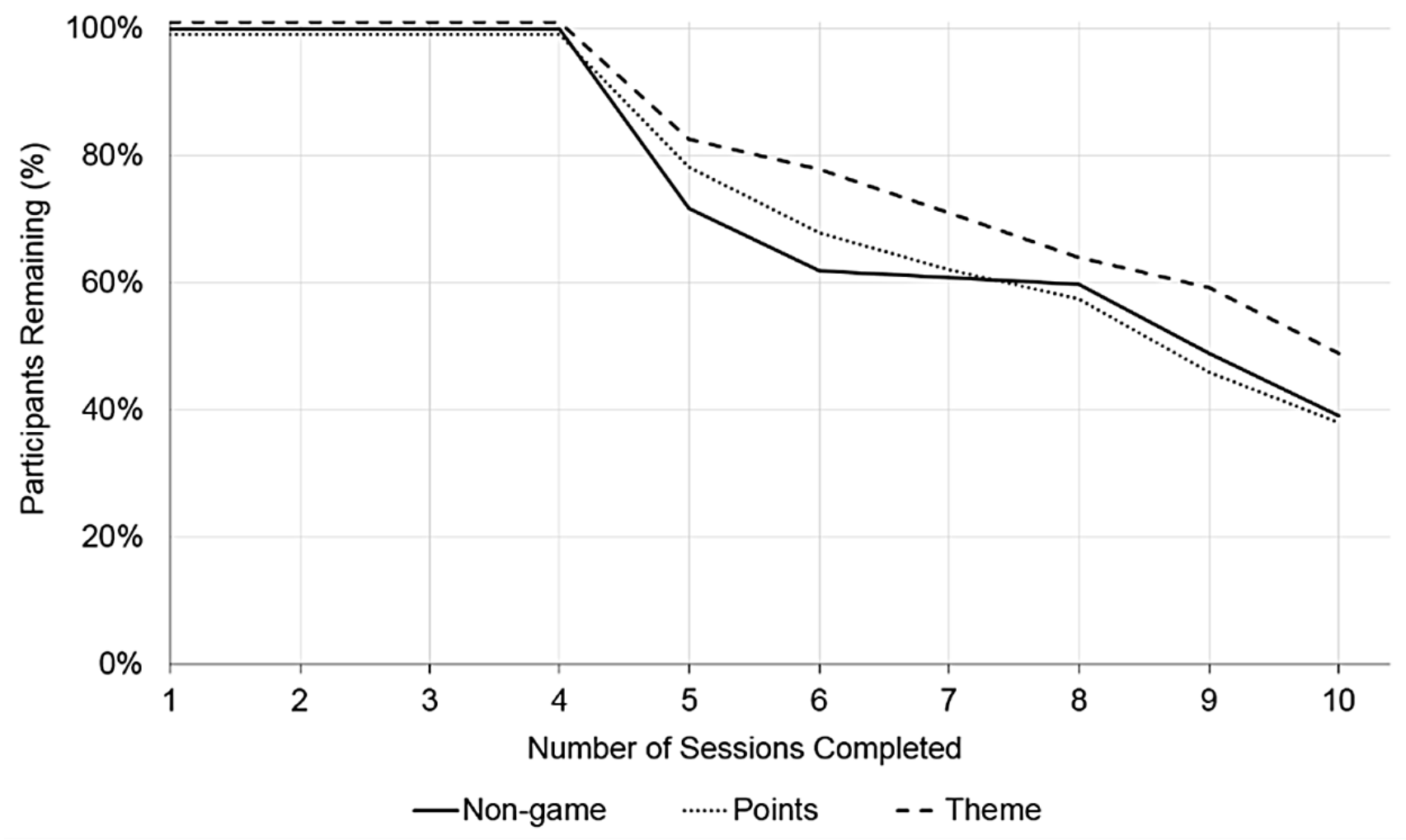


Figure 4: Overall scores from the subjective enjoyment and engagement questionnaire

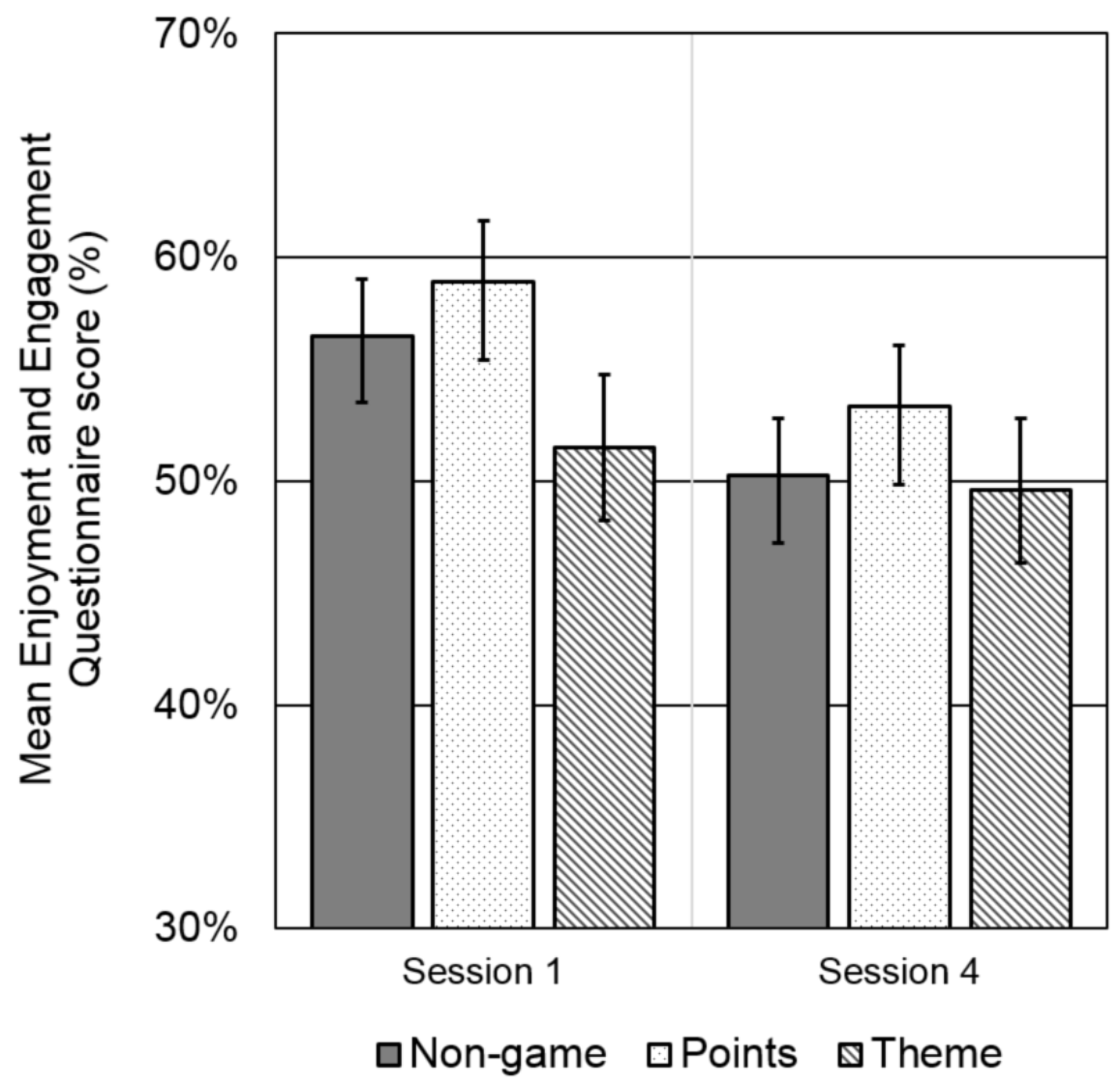

Mean responses of VAS scores from questionnaires delivered on sessions 1 and 4, shown separately by task variant and timepoint. Error bars represent $95 \%$ confidence intervals 
Figure 5: Boxplots of mean Stop Signal Reaction Time

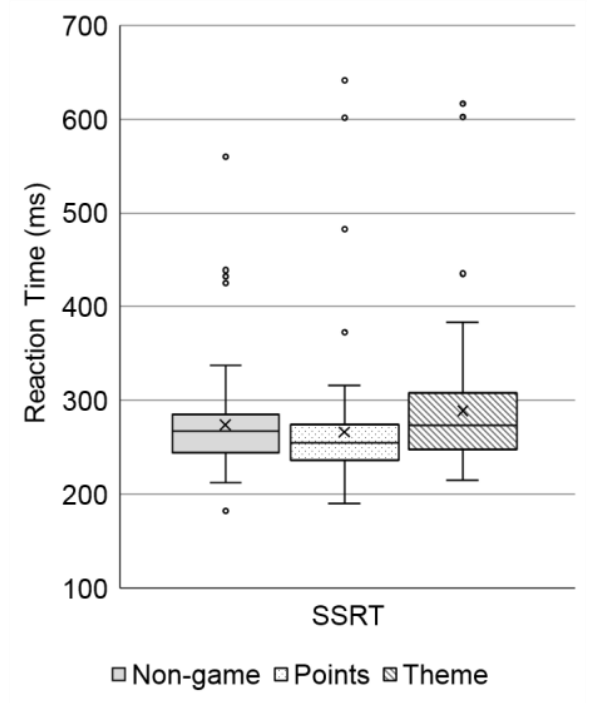

Data combined per participant over the first four sessions and shown separately by task variant 